Revista Calidad en la Educación Superior

Programa de Autoevaluación Académica

Universidad Estatal a Distancia, Costa Rica

ISSN 1659-4703

revistacalidad@uned.ac.cr

\title{
PRINCIPIOS CONSTRUCTIVISTAS PARA LA ELABORACIÓN DE UNA MEDIACIÓN PEDAGÓGICA APLICADA A PACIENTES CON ENFERMEDAD CARDIOVASCULAR
}

\author{
CONSTRUCTIVIST PRINCIPLES FOR THE DEVELOPMENT OF AN \\ EDUCATIONAL MEDIATION APPLIED TO CARDIOVASCULAR DISEASE \\ PATIENTS
}

\author{
Jorge Salas Cabrera ${ }^{1}$ \\ josaca10@hotmail.com \\ Universidad Nacional, Costa Rica
}

Volumen 6, número 1

Mayo 2015

Pp. 59 - 75

Recibido: 27 de noviembre, 2014

Aprobado: 19 de febrero, 2015

\footnotetext{
${ }^{1}$ Jorge Salas Cabrera, Doctor, académico de la Universidad Nacional, Escuela de Ciencias del Movimiento Humano y Calidad de Vida (CIEMHCAVI), Heredia, Costa Rica. Es coordinador del proyecto Rehabilitación Cardiaca Fase III.
} 


\section{Resumen}

Durante muchos años la rehabilitación cardiaca se ha convertido en un servicio de salud que facilita la recuperación de los pacientes con problemas cardiovasculares. Este servicio se encarga de estructurar un programa de ejercicio físico que involucra aspectos educativos de la forma saludable de alimentarse de cada persona, el apoyo social que se enfoca específicamente en la familia y amigos cercanos, el tiempo conveniente para mantener relaciones sexuales con su pareja y el control de situaciones que le generen estrés, depresión o ansiedad. Sin embargo, el abordaje que se realiza desde lo educativo se ha trabajado bajo el modelo conductual; el mismo provoca modificaciones en los hábitos de vida saludables a corto plazo y solo mientras se está en contacto permanente con la persona especialista. El propósito de este estudio es recopilar una serie de principios del modelo constructivista como herramientas para la elaboración de una mediación pedagógica aplicada a pacientes con enfermedad cardiovascular.

Palabras claves: constructivismo, rehabilitación cardiaca, pacientes cardiacos, educación, principios

\section{Abstract}

For many years, cardiac rehabilitation has become a health service which facilitates recovery of patients with cardiovascular problems, this service is responsible for structuring a program of physical exercise that also involves educational aspects including eating habits, social support, sexual and psychoemotional issues, However, the educational approach has been delivered using the behavioral model, which helps with short term changes in lifestyle habits and only while in constant contact with the specialist. The purpose of this study was to compile a set of principles of the constructivist model as tools for the development of a pedagogical mediation applied to patients with cardiovascular disease.

Keywords: constructivism, cardiac rehabilitation, cardiac patients, education, principles

El abordaje educativo que se le ha dado al proceso de la rehabilitación cardiaca ha sido, y continua siendo, de corte conductual. La consecuencia de esto es que las investigaciones en diferentes áreas educativas han permitido incrementar el conocimiento y facilitar modelos pedagógicos para un mejor aprendizaje. Estos modelos sirven de sustento teórico, y de ellos se derivan principios que ayudan a 
la población educadora en el proceso de mediación pedagógica en la dialéctica de la enseñanza o del aprendizaje (Palacios, 2000; Torres, 2008; Yampufé, 2009).

En el marco constructivista el uso de herramientas metodológicas orienta a la persona que aprende a transformar su propia realidad y también las representaciones que tiene de ella. Se parte del principio de que la persona es capaz de hacer una reconstrucción de su comprensión y de darse cuenta de su propia situación, rompiendo con viejos hábitos de vida o formas de actuar, haciendo conexiones entre los viejos y los nuevos conocimientos, lo cual permite generar un nuevo esquema mental (Vygotski, 1978; Counter, 2008; García y Cosseth, 2006; Sobrino, 2011).

Para dar sustento a la elaboración de una mediación pedagógica el modelo constructivista radical y cognitivo explica que el aprendizaje es responsabilidad de la persona, además que ella debe reflexionar acerca de la información que recibe, debe ser capaz de diferenciar los conocimientos previos y los nuevos, analizar y desarrollar un juicio crítico que le permita cambiar la actitud para cumplir con las intenciones educativas, tal como lo han señalado Corrales (2009) y Torres (2010). Por otro lado, estos modelos proponen que la persona que construye el conocimiento lo hace de forma activa y dinámica, interactuando con el entorno, modificando o especializando sus conocimientos con base en los conocimientos previos (Corrales, 2009; Torres, 2010; Rodríguez, 2011).

Con los aportes de los principios constructivistas la ejecución de una mediación pedagógica debería desarrollarse bajo situaciones en las cuales la persona transforme la manera de aprender, de tal forma que vaya diseñando y construyendo procesos de aprendizaje, convirtiendo el nuevo conocimiento en algo significativo, para que mediante los procesos cognitivos y metacognitivos logre resolver problemas de diversa índole (Hernández, 2008). 
El constructivismo describe los principios básicos del aprendizaje, concibiendo a la persona adulta como participante activa, apoyada en mediaciones pedagógicas que le permitan el establecimiento de relaciones en su vivencia cultural. Además, a partir de esta la persona logra generar una conexión con la nueva información, reestructurando y volviendo a organizar su pensamiento o sus estructuras cognitivas para atribuirle significado a lo nuevo. Si la persona construye ese conocimiento puede realizar modificaciones en su diario vivir, al asumir una posición crítica mediante el análisis y la reflexión sobre su propio comportamiento y sus experiencias de vida (Cubero, 2009; Kunoichisama, 2010).

En el proceso de recuperación que implica la enfermedad cardiovascular es necesario que se den cambios de comportamiento que impliquen un nuevo estilo de vida; en este contexto es preciso que el paciente cardiópata se entienda así mismo y a la patología que lo aqueja.

La persona, a través de una mediación pedagógica basada en un modelo constructivista, debe llevar a cabo un proceso de aprendizaje que le permita ser consciente y controlar sus acciones sin factores externos que las definan (Ferreiro, 2005; Dutan, 2007; Hernández, 2008; Counter, 2008; Corrales, 2009).

El propósito de este artículo es generar una reflexión sobre las posibilidades de mediación pedagógica derivadas de los modelos constructivistas en el proceso de aprendizaje y enseñanza aplicado a pacientes que asisten a los programas de rehabilitación cardiaca.

\section{Antecedentes del abordaje de la rehabilitación cardiaca}

A comienzos del siglo pasado la terapia a la que era sometida la persona intervenida quirúrgicamente por problemas cardiovasculares se basaba en guardar reposo en cama hasta por dos meses, para evitar una carga de trabajo cardiaco que pudiera tener consecuencias negativas. En este proceso normalmente se 
contaba con un enfermero encargado de asistir al paciente en las tareas cotidianas, entre ellas comer, beber, bañarse, arreglarse e incluso para darse vuelta en la cama (Linden, 1996).

En la década de los cuarenta, a partir de los aportes de Dock (1944) y Deitrick y Whedon (1948), se empezaron a estudiar los efectos que tenía la actividad física sobre el proceso de recuperación de la persona cardiópata. Estos autores llegaron a concluir que la práctica de actividad física unos días después de haber sufrido el infarto no ocasionaba ningún efecto perjudicial en la salud. Posteriormente, en 1952 se sientan las bases de la rehabilitación cardiaca, argumentando que la actividad física poco después de un evento cardiaco, aparte de reducir los efectos negativos del reposo, tenía efectos psicológicos y fisiológicos importantes. Esto da pie a que se empiece a pensar en la sistematización de programas de ejercicio físico estructurados, controlados y principalmente supervisados por diferentes especialistas en el campo de la promoción y rehabilitación de la salud cardiaca.

\section{Fases de la rehabilitación cardiaca}

Hellerstein y Ford (1957) fueron los primeros en poner en marcha un programa de rehabilitación cardiaca basado en el ejercicio físico y en el modelo vigente de tres fases. Fase I: la persona está hospitalizada (tiene una duración de quince días); fase II: es dada de alta y en convalecencia realiza actividad física de forma supervisada y monitorizada (tiene una duración de doce semanas); fase III: se encuentra en un proceso de recuperación e integración al trabajo (tiene una duración ilimitada, es decir, por el resto de la vida).

El aporte de Hellerstein y Ford (1957) fue visualizar la integración de pacientes cardiovasculares a la sociedad mediante un proceso de rehabilitación orientado hacia un enfoque multidisciplinario, es decir, bajo la supervisión de especialistas en el campo de la medicina, psicología, terapia física, fisiología del ejercicio y nutrición. Esta integración en el proceso de rehabilitación fue puesta en práctica 
también por Dressendorfer, Franklin, Cameron, Trahan, Gordon y Timmis (1995), quienes encontraron beneficios fisiológicos importantes en el perfil lipídico y en las respuestas funcionales de pacientes que asistían a los programas de rehabilitación cardiaca.

Sin embargo, todavía en estos programas de rehabilitación cardiaca se notaban debilidades en el funcionamiento, pues solo se atendía al paciente desde un enfoque biologista y de abordaje biomédico. Las intervenciones psicológicas y educativas solo mostraban resultados positivos mientras el paciente se encontrara en contacto directo con especialistas de cada área; sin embargo, una vez que mejoraban los aspectos fisiológicos y egresaban de los programas, volvían a las salas de atención de pacientes cardiacos, entre otras cosas a causa del abandono del ejercicio físico, debido a que asumían patrones de comportamiento poco saludables.

Numerosos estudios describen resultados positivos asociados a intervenciones integrales en las que se consideró lo psicológico, la actividad física y la educación en el caso de la persona paciente cardiaca (Cazorla, Fernández, Corcoy, Vidal, Tragant y Martínez, 2004; Hernández y Arranz, 2005).

\section{Modelo de intervención educativo existente para pacientes cardiacos}

Muchas de las intervenciones educativas en pacientes con enfermedad cardiovascular se han basado en metodologías conductistas (Hernández y Arranz, 2005; Mora, s. f.). Estos modelos fueron exitosos en la medida en la que el paciente modificaba sus conductas para llevar una vida con altos estándares de calidad; sin embargo, esta modificación no se presentaba a largo plazo por lo que era necesario dar un seguimiento al paciente para controlar su recuperación.

Los modelos que se han establecido a lo largo de los años en la rehabilitación cardiaca han fundamentado su quehacer bajo el enfoque conductual. Por ejemplo, en un estudio realizado por Solís, García, Casado, Zabala, Barragán, González, 
Millán (2005) se implementa un programa educativo para personas con trasplante de corazón. El resultado de difundir consejos útiles para pacientes en la aplicación de un programa educativo generó modificaciones importantes en sus hábitos de vida. Sin embargo, estos consejos fueron externados por enfermeros, es decir, utilizando una metodología conductual en la forma de trasmitir conocimiento nuevo entre especialista y paciente. Sigue existiendo deficiencia en el aprendizaje y en los cuidados que conlleva una rehabilitación cardiaca; por ello, es necesario que el abordaje pedagógico convierta en participante activa a la persona que tuvo problemas cardiacos, con el fin de que comprenda su patología.

Para elaborar una propuesta interesante y novedosa en la que se utilice una intervención diferente que trascienda el logro de resultados a corto plazo y ayude a educarse en aspectos como el autocuidado, la modificación de conductas y en la mejora de los estilos de vida, esto incluye la forma de pensar, emocionarse, relacionarse con los demás y de comportarse en relación al propio cuerpo. Estos cambios reducirán la probabilidad de un segundo evento cardiovascular. Este replanteamiento en el estilo de vida del paciente cardiópata requiere de una mediación pedagógica de carácter holista.

\section{Los modelos teóricos constructivistas}

Esta teoría establece que el aprendizaje es un proceso activo en el cual las personas, de manera particular, construyen nuevas ideas o conceptos basándose en su conocimiento previo. El paciente selecciona y transforma información, construye hipótesis y toma decisiones sobre la base de la estructura cognitiva actual. La estructura cognitiva (es decir, esquemas, modelos mentales) provee significado y permite la organización de las experiencias para que el individuo pueda ir más allá de la información y actividad que desea aprender (Ausubel y Novak, 1983). 
Por su parte, Von Glasersfeld (1995) manifestó que la actividad constructora de nuevo conocimiento corresponde necesariamente a situaciones que le permiten a la persona organizarse y a la vez organizar su mundo experiencial.

Según lo indicado por los dos autores, y para efectos de esta investigación, es preciso señalar que parte del problema que tienen las personas que siguen sufriendo patologías cardiacas es precisamente que no se les fortalece la capacidad para ser dinámicas en el procesamiento de la información educativa ni para modificar sus hábitos de vida, ya que presentan muchas deficiencias para organizarse y organizar dicha información; asimismo, en los programas de rehabilitación cardiaca se les induce a asumir una posición pasiva, recibiendo información sin la oportunidad de cuestionar, proponer u organizar estrategias para generar modificaciones en su comportamiento que ayuden en el proceso de rehabilitación.

Las teorías constructivistas plantean una medición pedagógica comprometida con las necesidades psicológicas del paciente para formar el aprendizaje. Es importante recordar que un $24 \%$ de los problemas relacionados con la enfermedad cardiaca provienen de aspectos emocionales, por lo que esta teoría promueve la identificación, motivación y atención de los intereses que cada persona tenga en el proceso de ir construyendo el aprendizaje de la nueva realidad. En el proceso de rehabilitación cardiaca es fundamental entender la manera en la que cada persona afronta la enfermedad respecto a sus intereses mediatos e inmediatos. Se trata de reforzar la motivación para que la persona busque y construya un nuevo conocimiento que le permita rehabilitarse y crecer como persona.

\section{La mediación pedagógica desde el modelo constructivista}

La mediación es una intervención que se practica para enriquecer la relación entre la persona y su ambiente (Cuenca, 2007). Durante el desarrollo de un proceso de mediación pedagógica es adecuado presentarle a la persona que aprende 
situaciones que sean significativas para ella. Esto facilita la adquisición de un conocimiento útil sobre propósitos claramente conscientes para la persona. La mediación pedagógica en el modelo constructivista obliga a la persona mediadora a tener claridad sobre la intencionalidad del proceso de superación de la enfermedad cardiaca. De manera que la buena comunicación entre pacientepersona mediadora es fundamental para un aprendizaje efectivo.

Desde el enfoque constructivista es importante tomar en cuenta todas las experiencias previas, la historia personal del enfermo más que el aquí y ahora. Esto favorece el involucramiento emocional y la disposición de las personas en el proceso de aprendizaje requerido para la interiorización de un estilo de vida que las proteja contra la patología cardiaca (Cuencas, 2007).

La mediación pedagógica debe favorecer el desarrollo de los sentimientos de competencia y logro para que la persona se sienta capaz y reconozca que este proceso le sirve para recuperar la salud. De esta manera se asegura la disposición necesaria para el aprendizaje y la aceptación de nuevos retos, lo cual le generará confianza y autoeficacia al paciente. Reforzar los sentimientos de seguridad y entusiasmo por aprender constituye la base sobre la que se construye una adecuada autoimagen. El reconocimiento de los logros y las habilidades a lo largo del proceso de rehabilitación induce al mejoramiento de la autoestima y fortalece el sentimiento de logro personal (Counter, 2008).

Desde la mediación pedagógica del modelo constructivista se espera que durante la construcción del nuevo conocimiento las personas adultas con enfermedad cardiaca adquieran conocimiento sobre su patología cardiovascular, para que durante el proceso se modifiquen aquellos comportamientos poco saludables en beneficio de la recuperación del paciente.

\section{Aprendizaje significativo desde el constructivismo}


El aprendizaje significativo es el resultado de la interacción entre las ideas principales existentes en la estructura cognitiva de la persona y la nueva información que se le brinda en el proceso de la rehabilitación, permitiendo una reestructuración cognitiva. Esto contribuye a una diferenciación, elaboración y estabilidad de los conocimientos previos existentes (Sánchez, Moreira y Caballero, 2011).

Si los conocimientos previos existentes en la estructura cognitiva son claros, estables y diferenciables se facilita la resolución de problemas. El aprendizaje significativo surge cuando la persona, como ser que construye su propio conocimiento, tiene la posibilidad de relacionar conceptos dándoles sentido a partir del conocimiento que ya posee. El aprendizaje se da cuando los contenidos están relacionados con algún aspecto existente en la vida de la persona, o se refieren al caso particular de una persona cardiaca (Sossa, Pavia y Ulisses, 2009).

Durante el desarrollo de la mediación pedagógica es indispensable que los comentarios realizados por los pacientes acerca de un contenido específico en el campo de la rehabilitación (alimentación, ejercicio, aspectos emocionales, sociales y conocimiento general acerca de la enfermedad) sean acordes con las experiencias vividas en algún momento, de manera que al plantear y construir el nuevo conocimiento reflexionen y se les facilite asimilar la nueva información haciéndola significativa.

En ese sentido, Ausubel y Novak manifestaron que «el aprendizaje del alumno depende de la estructura cognitiva previa que se relaciona con la nueva información, debe entenderse por "estructura cognitiva" al conjunto de conceptos, ideas que un individuo posee en un determinado campo del conocimiento, así como su organización» (1983, párrafo 6). 
Como se puede apreciar, de acuerdo a lo expresado por estos autores, en la medida en la que la persona establezca relaciones acerca de lo que aprende con su estructura cognitiva previa permite alcanzar un aprendizaje que le es significativo y que será recordado a lo largo de los años. Al depender de cada estructura cognitiva de la persona el tiempo en que se adquiera el conocimiento estará sujeto a la capacidad que tenga cada una en hacerlo significativo y la forma de relacionarlo con las ideas y los conceptos previos.

De manera que es importante que aquella persona que ha sufrido un problema cardiovascular establezca relaciones acerca de la enfermedad, sus causas y consecuencias, formas de cuidado y recuperación, de manera que el nuevo conocimiento alcanzado le signifique algo y logre modificar el comportamiento frente a la enfermedad.

En un estudio realizado por Blandino (2004) se encontró que los pacientes cardiópatas después de haber sido operados del corazón, en su mayoría, admitieron sentirse atemorizados por la amenaza de muerte, algunos consideraron que podían dominar este temor mejor que otros. Aquellos que no podían manejar bien ese sentimiento se sentían más perturbados, preocupados e inquisitivos sobre su tratamiento. Por otro parte, García y Cosseth (2006) realizaron un estudio en el que describieron y compararon las necesidades de aprendizaje y el sentimiento de autoeficacia en la gestión de factores de riesgo de la enfermedad coronaria. Los pacientes fueron sometidos a un proceso educativo durante la estancia en el hospital, en donde estudiaron aspectos como la anatomía, fisiología, actividad física, alimentación y factores de riesgo. En este estudio se comprobó que las personas consideran importante aprender sobre anatomía, fisiología y factores de riesgo. Con ello se mejoró la autoconfianza de los pacientes, base fundamental para generar cambios en el comportamiento que reduzcan las probabilidades de una patología cardiovascular. En resumen, para lograr un aprendizaje significativo desde el modelo constructivista la persona debe 
ir construyendo el nuevo conocimiento a partir del establecimiento de la información que se le brinde y las experiencias de vida, con sus creencias e ideas previas, de tal manera que esta construcción facilite que la persona adquiera un aprendizaje con sentido práctico.

\section{Aspectos epistemológicos en la educación de personas adultas y el papel del constructivismo}

El conocimiento no es más que una simple representación de la realidad externa; es el resultado de la interacción entre el sujeto que aprende (cognitivamente) y sus prácticas sensoriales. El sujeto adulto que aprende abandona la típica pasividad y construye, estructura sus experiencias, participa activamente en el proceso de forma real y verdadera (Moreno, 2010).

De acuerdo con Moreno «la educación para adultos se percibe como un proceso permanente e integral de formación y actualización, dirigido a la preparación de una persona crítica, democrática, dinámica, pero sobre todo tiene la intencionalidad de elevar su autoestima haciéndolo sentir productivo» (2010, p. 5). La epistemología del aprendizaje, para usar una terminología moderna, describe a una persona adulta dotada de un lenguaje desarrollado, con capacidad de abstracción y de generalización (D’Amore, 2009).

Las habilidades de aprendizaje conllevan modificaciones en la relación que la persona adulta mantiene con la realidad como objeto de conocimiento. Esta llega a percibir el conocimiento como una evolución social, activa de la realidad, a través de un proceso de reflexión crítico que tiene en cuenta el contexto histórico (Ruiz, 2001).

En ese sentido, la persona enferma del corazón o del sistema cardiovascular debe reconocer que es necesario aprender acerca de lo que representa la enfermedad cardiaca, sus características, consecuencias, la rehabilitación o la recuperación, 
de manera que, a partir de los procesos de reflexión crítica, construya estrategias que le faciliten la modificación de aquellos hábitos que sean poco saludables.

Los estudios de la andragogía (ciencia de la educación en personas adultas) enfatizan la importancia y la trascendencia que tiene elaborar teoría y praxis educativas que respondan a las características específicas, las necesidades, las limitaciones y las ventajas e intereses de las personas adultas (Alcalá, 2007).

La mediación pedagógica debe tomar como referencia las respuestas, los comentarios y las experiencias de vida de cada paciente, de tal forma que al construir el instrumento de mediación pedagógica el conocimiento nuevo permita que la persona genere reflexiones críticas y análisis eficaces, para lograr promover un cambio en el conocimiento y en la significancia para abordar su enfermedad de ahora en adelante.

\section{Conclusiones e implicaciones}

$>$ Los modelos educativos de intervención en pacientes con enfermedad cardiaca han enfocado sus objetivos en la enseñanza mediante la metodología conductual, por lo que se ha evidenciado que los pacientes logran cambios significativos a corto plazo; sin embargo, para lograr una rehabilitación cardiaca exitosa es necesario provocar cambios en el comportamiento de la persona que garanticen la interiorización de nuevos hábitos de vida saludables. El constructivismo promueve la participación activa de la persona en la comprensión a través del aprendizaje significativo a largo plazo.

> Las diferentes teorías que fundamentan los modelos constructivistas permiten elaborar mediaciones pedagógicas en las que se promueve la participación activa de la persona que aprende. En este caso, es el mismo paciente el que debe ir construyendo su propio conocimiento, relacionando sus ideas, pensamientos y experiencias previas con la nueva información que va 
adquiriendo; de esta manera la persona enferma del corazón va ir logrando modificar hábitos de vida al comprender la importancia de la rehabilitación cardiaca. Además, tal conocimiento debe ser significativo, de manera que sea permanente en el esquema mental del paciente.

> Bajo los principios constructivistas el uso de herramientas metodológicas orienta el aprendizaje a generar una transformación de la realidad del que aprende y de las representaciones que tiene de ella, logrando modificar comportamientos a través de la comprensión del nuevo conocimiento. La persona es capaz de hacer una reconstrucción del conocimiento y entender cómo se encuentra, rompiendo con viejos hábitos de vida o formas de actuar. Esto garantiza una rehabilitación cardiaca exitosa que le permite mejorar su calidad de vida.

\section{Referencias}

Alcalá, A. (2007). ¿Es la andragogía una ciencia? Recuperado de http://www.monografias.com/trabajos6/anci/anci.shtml.

Ausubel, D. y Novak, J. (1983). El mapa conceptual: una nueva herramienta de trabajo. Diseño de una práctica para fisiología. Recuperado dehttp://www.unizar.es/eees/innovacion06/COMUNIC_PUBLI/BLOQUE_ IV/CAP_IV_5.pdf

Blandino, J. (2004). Los factores que influyen en el Stress pre y post operatorio y las emociones. Universidad de la Tercera Edad, Santo Domingo.

Cazorla, M; Fernández, G: Corcoy, R; Vidal, C; Tragant, G; Martínez, A. (2004). Rehabilitación cardíaca. ¿Cómo mantener los cambios en el estilo de vida de nuestros pacientes. Recuperado de http://dialnet.unirioja.es/servlet/articulo?codigo=2331632 
Corrales, K. (10 de julio, 2009). Construyendo un segundo idioma, constructivismo y la enseñanza de L12. Revista Instituto de Estudios de la Educación, 10 (2), 156-167.

Counter, H. (2008). Constructivist Theories. Recuperado de http://mennta.hi.is/starfsfolk/solrunb/construc.htm

Cubero, F. (2009). Principios constructivistas y diversidad de la comunidad del aula. Recuperado de http://www.redires.net/?q=node/378

Cuenca, N. (2007). Didáctica constructivista como práctica mediadora para una cultura de paz. Universidad Pedagógica Experimental Libertador N. ${ }^{\circ} 3$.

D'Amore, B. (2009/11). Conceptualización, registros de representaciones semióticas y noética: Interacciones constructivistas en el aprendizaje de los conceptos matemáticos e hipótesis sobre algunos factores que inhiben la devolución. Revista Científica, 11, 150-164.

Deitrick, J. \& Whedon, G. (1948). Effects of mobilization upon various metabolic and Physiologic functions of normal men. American Journal of Medicine, 46(3), 3-35.

Dock, W. (1944). The evil sequelae of complete bed rest. Journal of the American Dorn, Naughton, Imamura, y Trevisan, № 125:1083-1085

Dressendorfer, R. H., Franklin, B. A., Cameron, J. L., Trahan, K. J., Gordon, S. y Timmis, G. C. (1995). Exercise training frequency in early post-infarction cardiac rehabilitation. Influence on aerobic conditioning. J. Cardiopulm Rehabil, 15(4), 269-276.

Dutan, H. (2007). Construcción del conocimiento como estrategia de desarrollo del conocimiento y saberes tácitos. Recuperado de http://www.gestiopolis.com/administracion-estrategia/construccion-delconocimiento-como-estrategia-de-desarrollo.htm

Ferreiro, R. (2005). El construtivismo social. Nova Southeaster University. Recuperado http://www.redtalento.com/Articulos/WEBSITE\%20Revista\%20Magister \%20Articulo\%206.pdf.

García, M. y Cosseth, S. (2006). Necesidades de aprendizaje y sentimiento de autoeficacia en los pacientes aquejados de un infarto de miocardio o de 
un angor. Libro de Ponencias. Encuentro de Investigación en Enfermería, Albacete, España.

Hernández, S. (2008). El modelo constructivista con las nuevas tecnologías: aplicado al proceso de Aprendizaje. Revista de Universidad y Sociedad del Conocimiento, 5(2), 26-35.

Hernández, D. y Arranz, H. (2005). Prevención y rehabilitación cardiaca. Generalidades. Recuperado de http://www.enfermeriaencardiologia.com/publicaciones/manuales/preve n/cap_01.pdf.

Hellerstein, H.K. Ford, A.B. (1957). Rehabilitation of the Cardiac Patient. American Heart Journal; 164:225.

Kunoichisama, K. (2010). El constructivismo en el aula. Recuperado de http://www.monografias.com/trabajos35/constructivismoaula/constructivismo-aula.shtml

Linden, W. (1996). Psychosocial Interventions for patients with Coronary Artery Disease. Archive International Medical, 156(8), 741-751.

Mora, J. A. (S. f.). Programas de prevención y rehabilitación cardiaca. Recuperado de http://www.enfermeriaencardiologia.com/publicaciones/manuales/preve n/cap_03.pdf

Moreno, S. (2010). Educacion en adultos (práctica social-opción de grado). Universidad Militar Nueva Granada,Colombia.

Palacios, M. A. (2000). La educación en América Latina y el Caribe; los procesos pedagógicos. Recuperado de http://www.schwartzman.org.br/simon/delphi/pdf/palacios.pdf

Rodríguez, E. (2011). Modelos Pedagógicos. Universidad Pedagógica de la Habana, Cuba.

Ruiz, C. (2001). ¿Cómo aprenden los adultos? Características del pensamiento. Revista Educación a Distancia, 1. Recuperado de http://revistas.um.es/red/article/view/25151/24421

Sánchez, I., Moreira, A. y Caballero, C. (2011). Implementación de una renovación metodológica para un aprendizaje significativo. Latin-American Journal Educ., 5(2), 475-484. 
Sobrino, A. (2011). Proceso de enseñanza-aprendizaje y web 2.0: valoración del conectivismo como teoría de aprendizaje constructivista. Estudios sobre educación, 20, 117-140.

Solís, M., García, F., Casado, M. J., Zabala, A., Barragán, A., González, T. y Millán, I. (2005). Programa de Educación para la Salud en el transplantado del corazón. Ensayo controlado con asignación aleatoria. Revista de Enfermería Clínica, 15(3), 131-140.

Sossa, C. A., Pavia, E. y Ulisses, J. U. (2009). Aptitud de enfermería en Pacientes con Paro Cardiorespiratorio: alcances de una estrategia educativa. Revista Enfermeria Instituto Mexicano Seguro Social, 17(2), 85-90.

Torres, M. J. (2008). Procedimiento didáctico en el proceso pedagógico para fortalecer el aprendizaje cooperativo de los estudiantes de 8 años de educación básica del colegio (tesis de licenciatura). Universidad Estatal de Bolívar, Ecuador.

Torres, M. G. (2010). Modelos Pedagógicos. Recuperado de: http://gingermariatorres.wordpress.com/modelos-pedagogicos/

Von Glasersfeld, E. (1995). Radical constructivism: A way of knowing and learning. Londres: The Falmer Press.

Vytgoski, L. (1978). Mind in society. Cambridge, MA.: Harvard University Press.

Yampufé, C. A. (2009). Los procesos pedagógicos en la sesión de aprendizaje. Recuperado de http://carlosyampufe.blogspot.com/2009/04/losprocesos-pedagogicos-en-la-sesion.html 